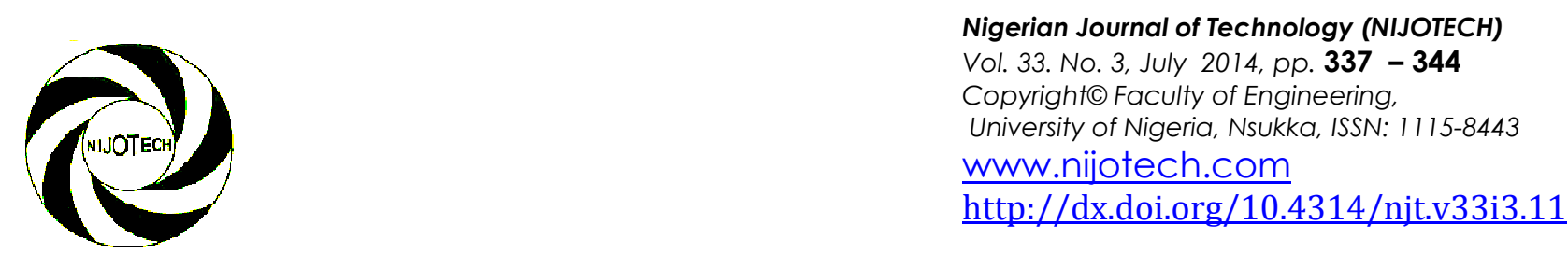

\title{
AN ALTERNATIVE APPROACH TO THE DETERMINATION OF OPTIMUM RESERVATIONS FOR HANDOVER IN GSM NETWORKS
}

\author{
M. O. Ezeja*

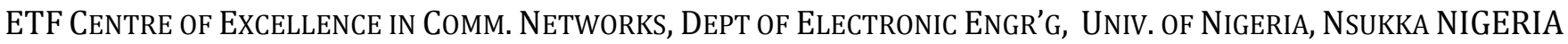 \\ E-mail address: obinna.ezeja@unn.edu.ng
}

\begin{abstract}
This work presents an alternative approach to the computation of the optimum reservations to be made in a GSM network for handover call requests. Prioritizing handover calls in a cellular network such as the GSM is necessary so as to guarantee seamless connection. Successful handover enhances the quality of service (QoS) of a network as the later is directly dependent on call continuity. In a previous analysis, the expression for the optimum reservations to be made was determined using the Gaussian Elimination method which had limitations because the power series used only considered the second order. Also, the set of equations to be solved was reduced to three thereby giving a result that does not quite represent the general system. In this work, the logarithmic approach is adopted which captures the total system capacity under test. A more reliable function for the determination of optimum reservations required in a system is thus derived. This new approach is also compared with that obtained using the Gaussian Elimination method.
\end{abstract}

Key words: GSM, resource reservation, quality of service, handover, call drop probability.

\section{INTRODUCTION}

In a typical cellular network, the radio and fixed links required are not permanently allocated for the duration of a call. Handover, which is also referred to as handoff in North America, is the switching of an ongoing call to a different channel or cell. It denotes the procedure that transfers an ongoing call from one cell to another as the user moves through the coverage area of a cellular system [1]. Handover has also been defined as a process of changing some of the radio parameters of a channel (frequency, time slot, spreading code, or combination of them) associated with an existing connection [2]. During a handover process, a cellular network automatically transfers a call from one radio channel to another radio channel while maintaining quality of services (QoS) of a call [3]. In every network, including GSM networks, traffic consists of user and signalling traffic [4]. One of the basic functions of the Radio Resource layer in the GSM architecture is the execution of handover as well as making measurements required for handover. In cellular network, both the mobile station and the Base Transceiver Station, (BTS) regularly measure the radio signal strength. The mobile station also transmits its measurements reports continuously to the BTS [5].

There are four categories of handover in the GSM system, which involve transferring a call within channels (time slots) in the same cell; cells (Base Transceiver Stations) under the control of the same Base Station Controller (BSC); cells under the control of different BSCs, but belonging to the same Mobile services Switching Center (MSC); and cells under the control of different MSCs. The first two types of handover, known as internal handovers, involve only one Base Station Controller (BSC). To save signaling bandwidth, they are managed by the BSC without involving the Mobile services Switching Center (MSC), except to notify it after the execution of the handover. The last two types of handover, known as external handovers, are handled by the MSCs involved in the communication process. A very important aspect of GSM is that the original MSC, the anchor MSC, remains responsible for most call-related functions, with the exception of subsequent inter-BSC handovers under the control of the new MSC, called the relay MSC [6]. 
In cellular mobile networks, the system design is such that the coverage region is divided into smaller cells in order to achieve high system capacity [7]. Each cell is covered by an individual base station [8]. The most serious problem that arises in this architectural design however, is the handover issue. This problem becomes more pronounced for high speed moving terminals where the handover rate increases and the probability that an ongoing call will be dropped due to the lack of a free traffic channel is high.

\section{REVIEW OF OTHER HANDOVER PROIRITIZATION SCHEMES}

Quite a number of mobility management schemes to handle handover issues have been developed and proposed. Li and Alfa [9], for example, studied the use of reserved channel schemes (RCS). In this scheme, the channels of each cell are divided into two sets. The first set of channels are called normal channels which serve both handover and new connections, while the second set of channels are called guard channels which are reserved for serving handover connections only [9]. Reserving guard channels for handover connections urges prudent consideration. Insufficient reservation implies that handover connections will compete for free channels with new connections and consequently leads to a higher handover blocking rate. On the other hand, prodigal reservation might increase the number of idle channels in a system. A channel is classified as an idle channel if it is reserved but has not been assigned to any connections. Obviously, new-connection blocking rate increases with the number of the idle channels. Thus, determining an appropriate number of guard channels for handover connections is an essential issue for handover management here.

Furthermore, Chua and Choo [10], studied and proposed the use of probabilistic channel reservation as a means of providing handover priority. The proposed probabilistic channel reservation (PCR) model operates in a fixed channel assignment environment where each cell is pre-assigned a fixed number of channels to support both new and handover calls. It is assumed that the network architecture permits each base station to know (or estimate) the probability that a call arriving in one of its adjacent cells will move into its cell at some point in time in the near future [10]. One way of realizing this is to have, within each cluster of cells in a given geographic region, a central control node monitor the user mobility patterns and calculate the handoff probabilities between every given pair of adjacent cells. The base stations in the cluster of cells are then informed of these handoff probabilities periodically by the central control node. Alternatively, a distributed approach in which each base station monitors the mobility patterns of users in its cell and calculates the handoff probabilities to each of its adjacent cell is also possible. In this case, base stations are periodically informed of the handoff probabilities by their adjacent peers [10].

Ebersman and Tonguz [11], in their work "handover ordering using signal prediction priority queuing in personal communication systems", proposed the use of queuing priority schemes (QPS). In this scheme, each base station provides a waiting queue for the mobile terminals with on-going connections, which enter a handover area from one of its adjacent cells. As there are available channels, a free channel will be assigned to a mobile terminal that is currently in the waiting queue. When the queue is found to be empty, the channel could be assigned to any mobile terminal which attempts to initiate a new connection [11].

Agrawal et al [12], proposed the use of preemptive and non-preemptive channel borrowing handover scheme. Channels in each cell are divided into two and pre-allocated for real-time and non real-time services. Usually, services in integrated wireless networks can be divided into two types which include real-time services (such as voice) and non-real-time services (such as data). To increase the channel utilization while keeping the quality of service (QoS) of each type of traffic, one type of service is allowed to borrow channels from the other under certain constraints. In the proposed scheme, only real-time service handover requests can borrow channels from non real-time channels. The channel utilization can be further enhanced if originating real-time service calls are also allowed to borrow channels [12].

Jin, et al [13] introduced a novel handover scheme. They presented a handover scheme called the intelligent channel reservation (ICR) scheme, which exploits the location prediction technologies to accurately reserve channel resources for handover connections to provide seamless connection environment for mobile terminals and applications. Bearing in mind the fact that each mobile terminal has its individual mobility characteristic, the ICR scheme utilizes a channel reserving notification procedure (CRNP) to collect adequate parameters for predicting the future location of individual mobile terminals. These parameters will be utilized by the handover 
prediction function to estimate the handover blocking rate and the number of idle channels. A cost function for calculating the damages from blocking the handover connections and idling channel resources, and a corresponding algorithm for minimizing the cost function are proposed based on the handover prediction estimations. Also, a guard channel decision maker (GCDM) determines the appropriate number of guard channels. The experimental results show that the ICR scheme does reduce the handover-blocking rate while keeping the number of idle channels small [13].

\section{MODELLING}

It is known that there are basically three modelling techniques including graphical modelling, computer simulation modelling, and analytical modelling. The later which involves mathematical analysis is used in this work to model a scheme that could reduce the call drop (handover failure) probability which in turn brings about improved quality of service. It has been shown that computer simulation and modelling has the advantage of ensuring greater flexibility and modelling accuracy [14]. Moreover, the performance of a communication network system revolves around efficient allocation of resources and thus resource allocation is a central issue in this work. The proposed scheme, the Resource Reservation Scheme (RRS), is modelled using the Markov birth-death process.

\section{THE MARKOV BIRTH-DEATH PROCESS}

To describe this process, assume there are ten free trunks (or channels) in a communication network at a particular time and a call comes and is served. Then, the number of trunks available is reduced to nine (death process). On the other hand, when served requests have finished using the channels and release them, the channels are free for use by other connection requests thereby increasing the number of available channels (birth process). This continuous increase and reduction in available resources in a network is referred to as a birth-death process.

The direction of movement is dependent on a probability associated with each of the states, $\mathrm{S}$ which is in turn dependent on the arrival rate, $\lambda$ and the service rate, $\mu$. The principle of equilibrium which in this case is defined by the fluid flow process states that flow-in is equal to flow-out. State equations are thus formed on this basis and the associated probabilities derived.

\section{THE PROPOSED MODEL}

The diagram in Figure 1 shows the physical and graphical representations of the proposed model.
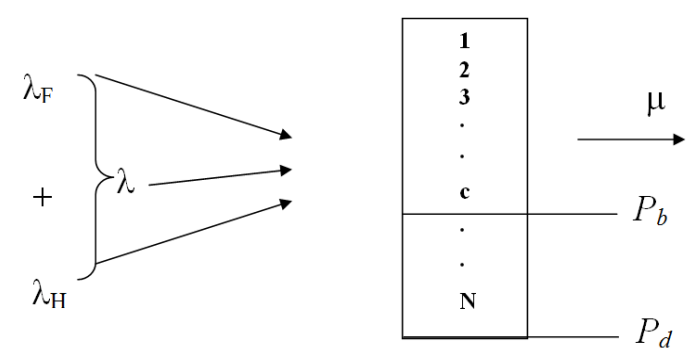

(a)

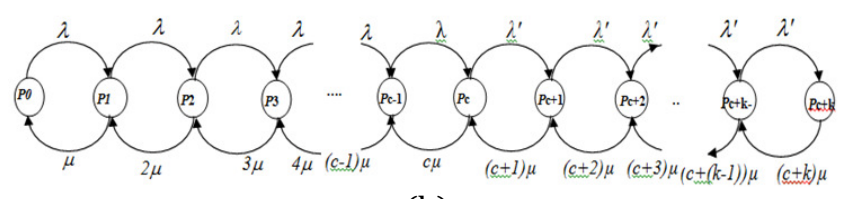

(b)

Figure 1: The Proposed Model (a) The physical representation (b) The graphical (Markov) representation

\section{DEFINITION OF PARAMETERS:}

$\lambda_{F}=$ arrival rate of the fresh calls - these are calls requesting for new connections and are also referred to as originating calls; $\lambda_{H}(=\lambda)=$ arrival rate of handover calls - these come from calls which are already served but require to handover to a new channel in the same cell due to weak signal or to a new channel in another cell due to its mobility as it crosses the cell boundary; $\lambda=$ combined arrival rate of both fresh calls and handover calls. In other words, $\lambda=\lambda_{F}$ $+\lambda_{H}$ (This is captured in the schematic diagram of the Physical Model); $\mathrm{k}=$ reserved resources; $\mathrm{c}=$ the number of resources that can serve both originating and handover call requests beyond which fresh calls can no longer be served; $\mu=$ service rate; $\rho=\lambda / \mu=$ traffic intensity of both fresh and handover calls; $\rho^{\prime}=$ $\lambda^{\prime} / \mu=$ traffic intensity of handover calls.

To develop the model, the state (fluid-flow) equations are:

$$
\begin{gathered}
S_{0}: \lambda P_{0}=\mu P_{1} \text { or } P_{1}=\frac{\lambda}{\mu} P_{0} \\
S_{1}: \lambda P_{1}+\mu P_{1}=\lambda P_{0}+2 \mu P_{2} \\
S_{2}: \lambda P_{2}+2 \mu P_{2}=\lambda P_{1}+3 \mu P_{3} \\
S_{3}: \lambda P_{3}+3 \mu P_{3}=\lambda P_{2}+4 \mu P_{4} \\
S_{c-1}: \lambda P_{c-2}+c \mu P_{c}=\lambda P_{c-1}+(c-1) \mu P_{c-1} \\
S_{c}: \lambda^{\prime} P_{c}+c \mu P_{c}=\lambda P_{c-1}+(c+1) \mu P_{c+1} \\
S_{c+1}: \lambda^{\prime} P_{c+1}+(c+1) \mu P_{c+1} \\
=\lambda^{\prime} P_{c}+(c+2) \mu P_{c+2} \\
S_{c+2}: \lambda^{\prime} P_{c+2}+(c+2) \mu P_{c+2} \\
=\lambda^{\prime} P_{c+1}+(c+3) \mu P_{c+3}
\end{gathered}
$$


$S_{c+(k-1)}: \lambda^{\prime} P_{c+(k-1)}+(c+(k-1)) \mu P_{c+(k-1)}$

$$
=\lambda^{\prime} P_{c+(k-2)}+(c+k) \mu P_{c+k}(9)
$$

$S_{c+k}: \lambda^{\prime} P_{c+(k-1)}=(c+k) \mu P_{c+k}$

Solving recursively, substituting equation (1) into equation (2) yields:

$\lambda\left(\frac{\lambda}{\mu}\right) P_{0}+\mu\left(\frac{\lambda}{\mu}\right) P_{0}=\lambda P_{0}+2 \mu P_{2}$

or

$P_{2}=\frac{\lambda^{2}}{2 \mu \cdot \mu} P_{0}=\frac{1}{2}\left(\frac{\lambda}{\mu}\right)^{2} P_{0}$

Substituting equation (1) and equation (11) into equation (3) yields:

$P_{3}=\frac{1}{3 \mu} \cdot \frac{\lambda^{3}}{2 \mu^{2}} P_{0}=\frac{1}{6}\left(\frac{\lambda}{\mu}\right)^{3} P_{0}$

Substituting equations (1), (11) and (12) into (4), we have:

$P_{4}=\frac{1}{4 \mu} \cdot \frac{\lambda^{4}}{6 \mu^{3}} P_{0}=\frac{1}{24}\left(\frac{\lambda}{\mu}\right)^{4} P_{0}$

Hence we observe that:

$P_{i}=\frac{1}{i !}\left(\frac{\lambda}{\mu}\right)^{i} P_{0}$

$P_{c}=\frac{1}{c !}\left(\frac{\lambda}{\mu}\right)^{c} P_{0}$

Now, $\quad \frac{\lambda}{\mu}=\rho=$ total traffic intensity. Hence,

$P_{c}=\frac{\rho^{c}}{c !} P_{0}$

Beyond state $S_{c}$ a new arrival rate $\lambda^{\prime}\left(=\lambda_{\mathrm{H}}\right)$, is defined for handover calls only, which can be served by $\mathrm{k}$ reserved resources. Hence the state equations from that state are (from equation 6):

$S_{c}: \lambda^{\prime} P_{c}+c \mu P_{c}=\lambda P_{c-1}+(c+1) \mu P_{c+1}$

Now, $P_{1}=\frac{\lambda}{\mu} P_{0} ; P_{2}=\frac{\lambda}{2 \mu} P_{1} ; P_{3}=\frac{\lambda}{3 \mu} P_{2}$ etc

$P_{c}=\frac{\lambda}{c \mu} P_{c-1} \quad \rightarrow \quad c \mu P_{c}=\lambda P_{c-1}$

Substituting into equation (6) yields:

$\lambda P_{c}=(c+1) \mu P_{c+1}$

Or $P_{c+1}=\frac{1}{(c+1) \mu} \lambda P_{c}$

From equation (7),

$\frac{\lambda^{\prime} \lambda^{\prime} P_{c}}{(c+1) \mu}+\frac{(c+1) \mu \lambda^{\prime} P_{c}}{(c+1) \mu}=\lambda^{\prime} P_{c}+(c+2) \mu P_{c+2}$

Or $\frac{\left(\lambda^{\prime}\right)^{2}}{(c+1) \mu} P_{c}+\lambda^{\prime} P_{c}=\lambda^{\prime} P_{c}+(c+2) \mu P_{c+2}$

$P_{c+2}=\frac{1}{(c+2) \mu} \frac{\left(\lambda^{\prime}\right)^{2}}{(c+1) \mu} P_{c}$

$=\frac{1}{(c+2)(c+1)}\left(\frac{\lambda^{\prime}}{\mu}\right)^{2} P_{c}$
Also from equation (8),

$$
\begin{aligned}
& \lambda^{\prime} \frac{1}{(c+2)(c+1)}\left(\frac{\lambda^{\prime}}{\mu}\right)^{2} P_{c}+(c \\
& +2) \mu \frac{1}{(c+2)(c+1)}\left(\frac{\lambda^{\prime}}{\mu}\right)^{2} P_{c} \\
& \quad=\left(\frac{\lambda}{(c+1) \mu}\right) \lambda P_{c}+(c+3) \lambda P_{c+3} \\
& \rightarrow P_{c+3}=\frac{1}{(c+3) \mu} \frac{\left(\lambda^{\prime}\right)^{3}}{(c+2)(c+1) \mu^{2}} P_{c} \\
& =\frac{1}{(c+3)(c+2)(c+1)}\left(\frac{\lambda^{\prime}}{\mu}\right)^{3} P_{c}
\end{aligned}
$$

Hence,

$P_{c+k}=\prod_{n=1}^{k}\left(\frac{1}{c+k}\right)\left(\frac{\lambda^{\prime}}{\mu}\right)^{k} P_{c}=\frac{c !}{(c+k) !}\left(\frac{\lambda^{\prime}}{\mu}\right)^{k} P_{c}$

Generally therefore,

$P_{c+n}=\frac{c !}{(c+n) !}\left(\frac{\lambda^{\prime}}{\mu}\right)^{n} P_{c}$

Recall the boundary condition:

$\sum_{i=0}^{\infty} P_{i}=1$

Or $\sum_{i=0}^{c} \frac{1}{i !}\left(\frac{\lambda}{\mu}\right)^{i} P_{0}+\sum_{n=1}^{k} \frac{c !}{(c+n) !}\left(\frac{\lambda^{\prime}}{\mu}\right)^{n} P_{c}=1$

Substituting the definitions of the parameters and equation (15) into equation (22) yields

$\sum_{i=0}^{c} \frac{1}{i !}(\rho)^{i} P_{0}+\sum_{n=1}^{k} \frac{c !}{(c+n) !}\left(\rho^{\prime}\right)^{n} \frac{1}{c !} \rho^{c} P_{0}=1$

Or $\sum_{i=0}^{c} \frac{\rho^{\prime}}{i !} P_{0}+\sum_{n=1}^{k} \frac{c !}{(c+n) !}\left(\rho^{\prime}\right)^{n} \frac{\rho^{c}}{c !} P_{0}=1$

Thus, $P_{0}\left[\sum_{i=0}^{c} \frac{\rho^{\prime}}{i !}+\sum_{n=1}^{k} \frac{c !}{(c+n) !}\left(\rho^{\prime}\right)^{n} \frac{\rho^{c}}{c !}\right]=1$

which implies that:

$P_{0}=\left[\sum_{i=0}^{c} \frac{\rho^{\prime}}{i !}+\sum_{n=1}^{k} \frac{c !}{(c+n) !}\left(\rho^{\prime}\right)^{n} \frac{\rho^{c}}{c !}\right]^{-1}$

(This is the probability of having a $100 \%$ resource availability and is associated with state, So. This is the state at which any call that arrives finds the system completely idle. In other words, this first call is assured of service).

Therefore, the call blocking probability for originating/fresh calls, $\mathrm{P}_{\mathrm{c}}$ is obtained by substituting this value into equation (16) as:

$P_{c}=\frac{\rho^{\prime}}{c !}\left[\sum_{i=0}^{c} \frac{\rho^{\prime}}{i !}+\sum_{n=1}^{k} \frac{c !}{(c+n) !}\left(\rho^{\prime}\right)^{n} \frac{\rho^{c}}{c !}\right]^{-1}$ 
Also, the call dropping probability for handoff calls, $\mathrm{P}_{\mathrm{d}}$ is obtained by substituting $\mathrm{P}_{\mathrm{c}}$ into equation (20) as:

$$
\begin{aligned}
P_{d}=\frac{c !}{(c+k) !}\left(\rho^{\prime}\right)^{k} \frac{\rho^{c}}{c !}\left[\sum_{i=0}^{c} \frac{\rho^{\prime}}{i !}\right. \\
\left.+\sum_{n=1}^{k} \frac{c !}{(c+n) !}\left(\rho^{\prime}\right)^{n} \frac{\rho^{c}}{c !}\right]^{-1}
\end{aligned}
$$

\section{VALIDATION OF THE PROPOSED MODEL}

The diagram in Figure 2 shows the physical and graphical representations of the proposed model. To test the validity of the proposed Resource Reservation Model (RRM), Lee's queuing model and the reservation model were both subjected to the same conditions and their behaviours compared. It was found as detailed below that both models behaved exactly the same way. We set both the number of queue spaces provided and the number of reserved resources in a system to zero, (that is, M1 $=0$; M2 = 0 ; and $\mathrm{k}=0$;) and arbitrary values were assigned to the other parameters as follows: arrival rate, $\mathrm{a}=\rho=$ 0.5 , System Capacity (Maximum Number of Resources), $\mathrm{N}=20$.

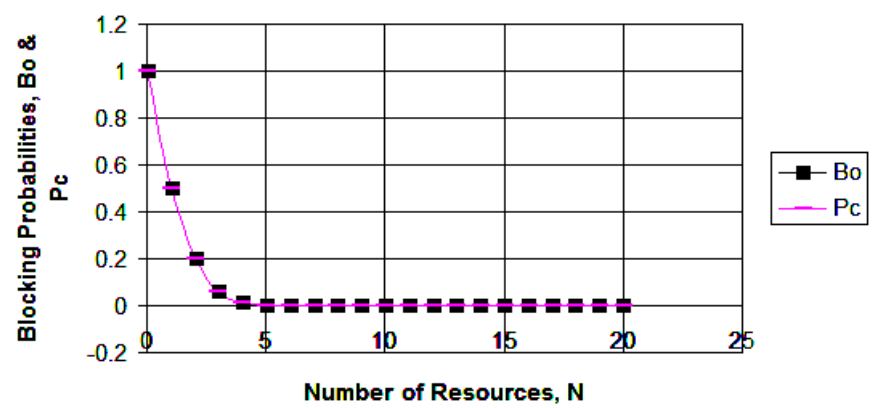

Figure 2: Call Blocking Probabilities for the Queuing Model, Bo and the Reservation Model, PC.

It can be seen from Figure 2 that when the number of resources in a system is zero (i.e. $\mathrm{N}=0$ ), the call blocking probabilities for both the Queuing model (represented with Bo) and that for the Reservation model (represented with Pc) are unity. With $\mathrm{N}=2$, the blocking probabilities become 0.5 for both models. It is also observed that this trend continues till the point at which $\mathrm{N}=20$. This implies that the rates of reduction in the probability of a call being blocked as the number of resources increase are exactly the same for both models.

\section{RESULTS FROM THE RESOURCE RESERVATION MODEL (RRM).}

The approach adopted in testing the model was to observe and record the behaviour (that is, change in the call blocking probabilities, Pc, for the fresh calls and change in call dropping probabilities, $\mathrm{Pd}$, for handover calls) of the model for varying number of reserved resources, $\mathrm{k}$ in a system. The traffic intensities $\rho$ and $\rho^{\prime}$ as previously defined were assigned values of 0.9 and 0.7 respectively. The behaviour of the model was then noted and recorded for number of resources, $N=10,15,20,25$, and 30 respectively. The results are as shown below:

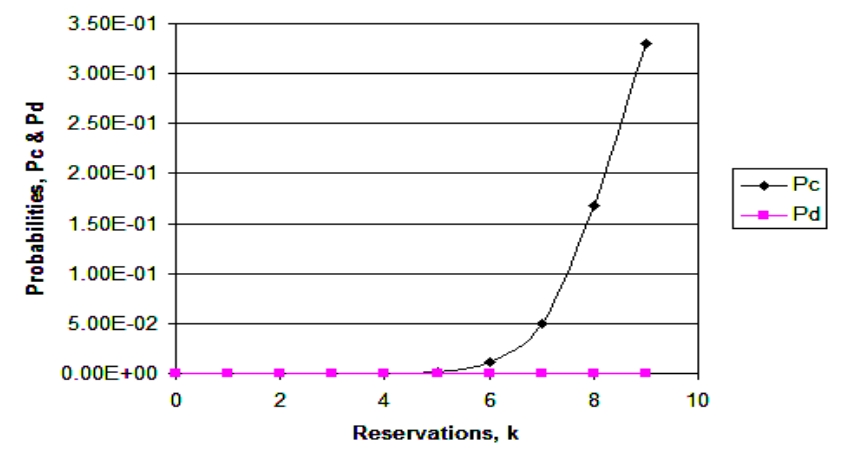

Figure 3: Call Blocking and Call Dropping Probabilities, $P c$ and $P d$ versus Reservations, $k(N=10)$

From Figure 3 showing the behaviour of the model with respect to the call blocking probability, Pc and the call dropping probability, $\mathrm{Pd}$, it is obvious that for zero reservation $(k=0)$, both Pc \& Pd have a value of 3.91E-8. This is expected because at this point, no reservation is made yet for the handover calls hence both fresh and handover calls should have the same chance of being served or blocked. With $\mathrm{k}=1$, the values change as Pc slightly increases to $4.34 \mathrm{E}-7$ and Pd slightly decreases to 3.04 E-8. Further reservations continue to show progressive decrease in $\mathrm{Pd}$ and a simultaneous progressive increase in Pc. However, it is observed that beyond four reservations, $(\mathrm{k}=4)$, there is a significant increase in Pc. This becomes the optimum reservations, $\mathrm{R}$ for $\mathrm{N}=10$. Note that the points appear to be zero progressively only because of the very gradual manner in which call drop probabilities change.

From Figure 4, it is obvious that for zero reservation $(\mathrm{k}=0)$, both Pc \& Pd have a value of $6.4 \mathrm{E}-14$. With $\mathrm{k}$ $=1$, the values change as Pc slightly increases to 1.07 E-12 and Pd slightly decreases to 4.98 E-14. Further reservations continue to show progressive decrease in $\mathrm{Pd}$ and a simultaneous progressive increase in Pc. However, it is observed that beyond nine reservations, 
$(\mathrm{k}=9)$, there is a significant increase in Pc. This becomes the optimum reservations, $\mathrm{R}$ for $\mathrm{N}=15$.

It is also clear from the Figure 5, that for zero reservation $(\mathrm{k}=0)$, both Pc \& Pd have a value of 2.03 E-20. With $\mathrm{k}=1$, the values change as Pc slightly increases to $4.51 \mathrm{E}-20$ and Pd slightly decreases to $1.58 \mathrm{E}-20$. Further reservations also as in other cases continue to show progressive decrease in $\mathrm{Pd}$ and a simultaneous progressive increase in Pc. However, it is observed that beyond thirteen reservations, $(\mathrm{k}=$ 13), there is a significant increase in Pc. This becomes the optimum reservations, $\mathrm{R}$ for $\mathrm{N}=20$.

It is also easily seen from Figure 6 that for zero reservation $(\mathrm{k}=0)$, both $\mathrm{Pc} \& \mathrm{Pd}$ have a value of $1.88 \mathrm{E}-27$. With $\mathrm{k}=1$, the values change as Pc slightly increases to $5.23 \mathrm{E}-26$ and Pd slightly decreases to $1.46 \mathrm{E}-27$. Further reservations also as in other cases continue to show progressive decrease in Pd and a simultaneous progressive increase in Pc. However, it is observed that beyond eighteen reservations, $(\mathrm{k}=$ 18), there is a significant increase in Pc. This becomes the optimum reservations, $\mathrm{R}$ for $\mathrm{N}=25$.

It is also easily seen from Figure 7 that for zero reservation $(\mathrm{k}=0)$, both Pc \& Pd have a value of 6.50 E-35. With $\mathrm{k}=1$, the values change as Pc slightly increases to $2.17 \mathrm{E}-33$ and Pd slightly decreases to $5.05 \mathrm{E}-35$. Further reservations also as in other cases continue to show progressive decrease in Pd and a simultaneous progressive increase in Pc. However, it is observed that beyond twenty-four reservations, ( $\mathrm{k}$ $=24$ ), there is a significant increase in Pc. This becomes the optimum reservations, $\mathrm{R}$ for $\mathrm{N}=30$.

\section{SUMMARY OF OBSERVATIONS AND ANALYSIS OF RESULTS}

The following observations were made and the implications are as given:

- The actual values of Pc and Pd are influenced by the choice of the arrival rate, $\lambda$ and the service rate, $\mu$. The ratio $\lambda / \mu$ is however used in this work and it represents the traffic intensity, $\rho$.

- When there is no reservation of resources for handover calls (that is $\mathrm{k}=0$ ), the two categories of connection requests, the fresh calls and the handover calls, compete for channels with equal chances of being served. This is expected because with no priority given to handover calls, the call blocking probability should be the same for both classes of calls.

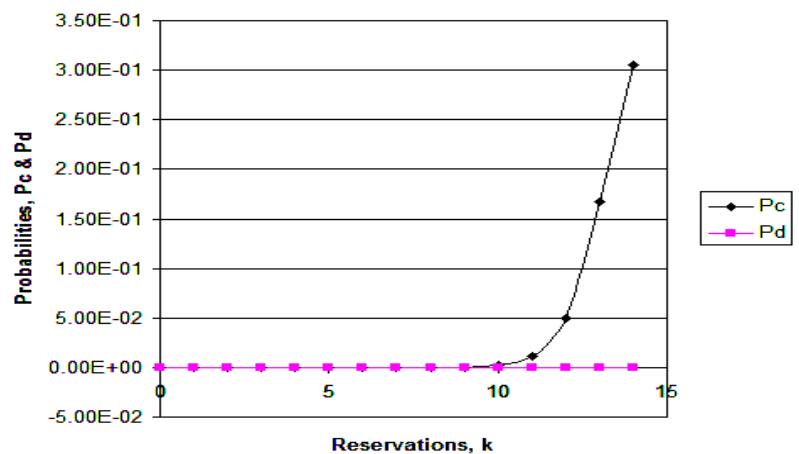

Figure 4: Call Blocking and Call Dropping Probabilities, $P c$ and Pd versus Reservations, $k(N=15)$

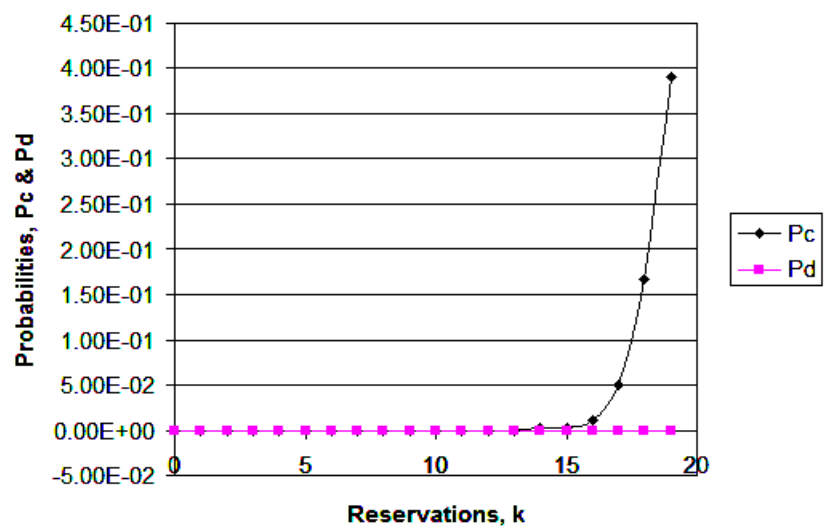

Figure 5: Call Blocking and Call Dropping Probabilities, $P c$ and Pd versus Reservations, $k(N=20)$

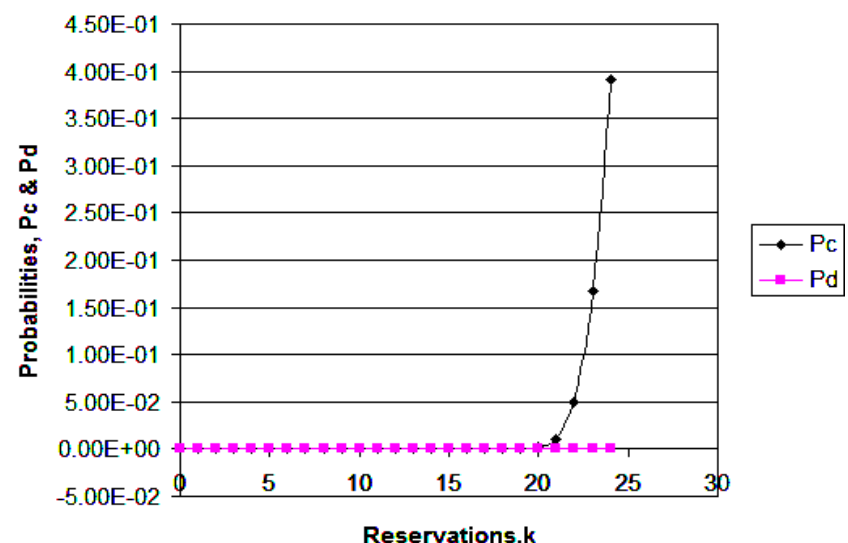

Figure 6: Call Blocking and Call Dropping Probabilities, $P c$ and Pd versus Reservations, $k(N=25)$

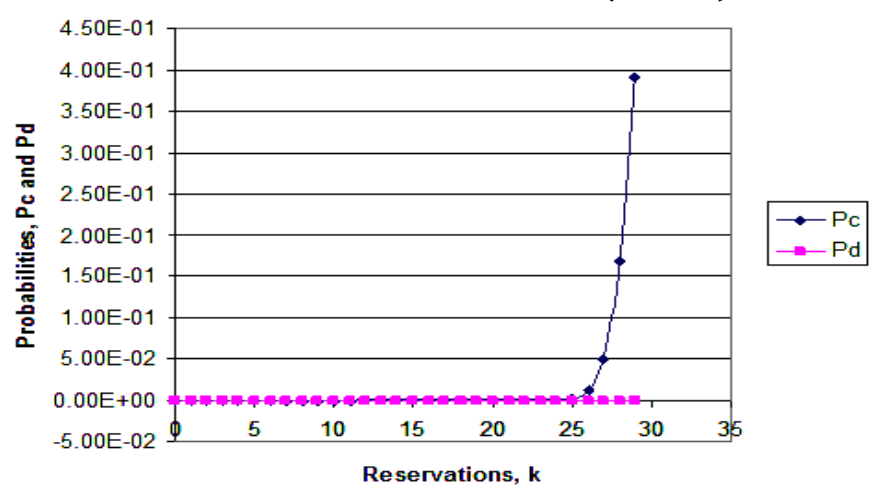

Figure 7: Call Blocking and Call Dropping Probabilities,

$P c$ and $P d$ versus Reservations, $k(N=30)$ 
- From figure 4 , with $\mathrm{N}=10$, the number of reservations is 4 (that is the point at which the fresh call blocking probability begins to rise significantly). From figure 5 , the number of reservations is 9 with $\mathrm{N}=15$. Also, with $\mathrm{N}=20$ in figure 6, we have 13; from figure 7 with $\mathrm{N}=$ 25 , we have 18 and with $N=30$ in figure 8 , we have 24 .

- These numbers tend to increase with increasing number of resources because from literature, handover call requests (which should be given priority), arrive in the system at an alarming rate and in a much higher proportion than the fresh calls. Firstly, handover calls come from mobile stations crossing their cell boundary into another cell due to its mobility (inter-system handover requests). Secondly, handover requests come from mobile stations in points of weak signal below a specified threshold within the cell referred to as hole (intra-system handover requests). These requests are made to a Base Station Controller (BSC) asking for a change of channel. Thirdly, the MSC may find that some cells are too congested while others are not. Then it would request cell sites to create early handovers for those congested cells. The wired infrastructure (MSC, BSC) may decide that the traffic in one cell is too high and shift some mobile stations to other cells with a lower load (referred to as load balancing). Moreover, Jochen Schiller had noted that about forty reasons for handover have been identified in the GSM standard [15].

- Once a single space is reserved for handover calls, the call drop probability, Pd is seen to improve (that is decrease) while the call blocking probability, $\mathrm{Pb}$ increases slightly. It is important to recall however that from literature, handover protection and prioritization strategies incur negative impact on the new user admission.

- Further increase in reservations results in increased improvement albeit at the expense of new call admission.

- Unlike the queuing model, the improvement in call drop probability in this reservation model continues indefinitely. This is desirable as it allows the network system designer to define the tradeoff point. In other words, it gives the designer the chance to choose what level of fresh call blocking he is willing to accept in order to obtain a particular quality of service (which is a function of handover success rate).

\section{DETERMINATION OF THE OPTIMUM RESERVATIONS IN A SYSTEM}

The optimum number of resources to be reserved for handover calls in any system can be estimated by taking a close look at the results obtained above and carrying out a mathematical analysis. The optimum reservation, $\mathrm{R}$ was determined and found to be 4, 9, 13,18 , and 24 for each of the system capacities, $\mathrm{N}=$ $10,15,20,25,30$ respectively. Then these values were plotted and a curve was obtained as shown in Figure 8.

To determine the expression for the maximum reservations, $\mathrm{R}$, to be made for a given network with system capacity, $\mathrm{N}$, we find the logarithmic expression representing the trend shown in the plot (Figure 8) obtaining a logarithmic function of the curve as shown. Hence, the relationship between the optimum reservations in a system, $\mathrm{R}$, and the System Capacity ( Number of Resources), $\mathrm{N}$, is as shown below:

$R=17.50 \ln (N)-37.61$

The Gaussian method adopted before had two limitations: (1) it limited the range of system capacities, $\mathrm{N}$ to only three out of the five under test for easy/less complex computation; (2) it also reduced the order of the resultant polynomial to 2 during the derivation resulting in a quadratic expression (which consequently represents the expression using the approach) given as:

$N=7.0008+0.6386 R+0.0278 R^{2}$

\section{COMPARISON BETWEEN THE GAUSSIAN APPROACH AND LOGARITHMIC APPROACH}

The performance of the expressions obtained using Gaussian Elimination method and the Logarithmic approach were evaluated. The simulation was done with Microsoft Excel as the system capacity was arbitrarily assigned values $\mathrm{N}=10,15,20,25,30,35$, and 40. The corresponding Optimum Reservations, $\mathrm{R}$ using the different expressions were obtained and plotted against the System Capacities, N. The result is shown in Figure 9.

Apparently, the two approaches yielded similar Optimum Reservations, $\mathrm{R}$ for a given system with capacity, N with slight variations. The optimum reservation curve from the logarithmic approach, $\mathrm{R}$ (Log) produced a better curve in that it took into consideration the entire system capacity range unlike 
the Gaussian method which only considered 3 out of 5 different system capacities. In addition, R (Log) has slightly higher reservations for handover requests in a system as seen from the graphs.

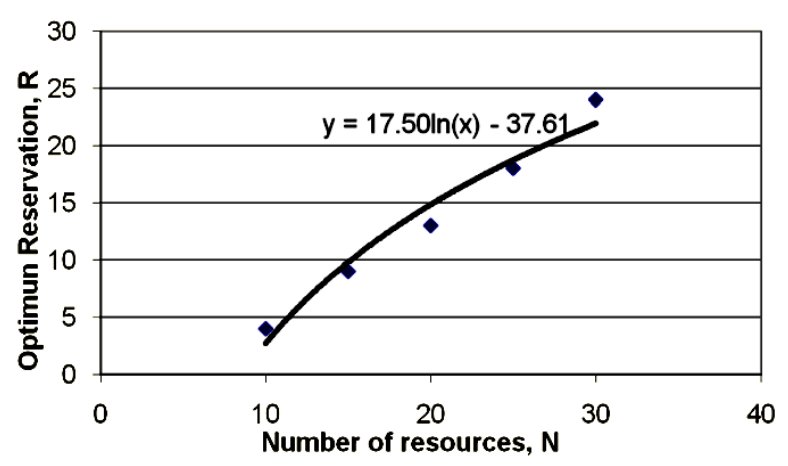

Figure 8. Logarithmic plot of Optimum Reservation versus Number of Resources, $N$.

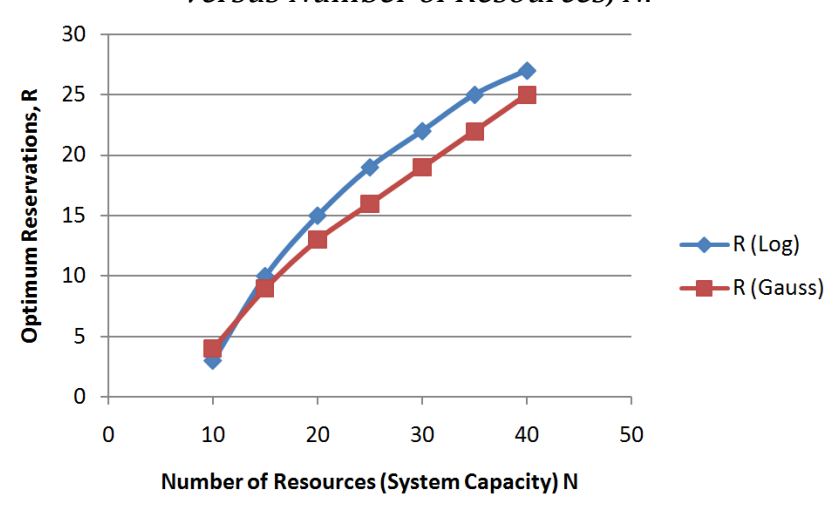

Figure 9: Comparison between $R$ (Log) and R (Gauss)

\section{CONCLUSION}

In this paper, the expression for the optimum number of resources to be reserved, $\mathrm{R}$ for handover call connections in a GSM network of a given system capacity $\mathrm{N}$, was derived. This expression for handover call ensures call continuity which in turn guarantees acceptable quality of service (QoS). The expression captures the trend in the entire scenario under test as it does not limit the work to any order. Secondly all the points in the curve are captured to truly represent the behavior in terms of response to handover success for given number of reservations in the system.

\section{REFERENCES}

[1] Jahangir K., "Handover management in GSM cellular system". International Journal of Computer Applications, Volume 8- No.12, October 2010.

[2] Ioannou, K., Louvros, S., Panoutsopoulos I., Kotsopoulos, S., and Karagiannidis, G. K., 'Optimizing the Handover Call Blocking Probability in Cellular Networks With High Speed Moving
Terminals" IEEE Communication Letters., vol. 6 no. 10. Oct., 2002, p. 1

[3] Chi Wan Sung, Member, IEEE "Analysis of Fade Margins for Soft and Hard Handoffs in Cellular CDMA Systems" IEEE Transactions on Wireless Communications. vol. 2, no. 3. May 2003 p. 432.

[4] Ani, C. I. GSM network traffic analysis, Nigerian Journal of Technology, Vol. 29, No. 2, June 2010, pp.54.

[5] Jahangir Khan et al, "Cellular Handover Approaches in 2.5G to 5G Technology", International Journal of Computer Applications Volume 21, No.2, May 2011

[6] John Scouriars. Overview of the Global Systems for Mobile Communication. http://jscouria@www. shoshin.uwaterloo.ca

[7] Bhuvaneswari, A., George, E. and Raj, D. P. Survey on Handoff Techniques. Journal of Global Research in Computer Science Volume 2, No. 6, June 2011, p. 140.

[8] Pollini, G. P. "Trends in Handover Design", IEEE Communications Magazine, March 1996, pp. 82 90.

[9] Li W. and Alfa A. S., "Channel reservation for handoff calls in a PCS network," IEEE Trans. Veh. Technol., vol. 49, Jan. 2000, pp. 95-104.

[10] Kee Chaing Chua and S. Y. Choo, "Probabilistic Channel Reservation Scheme for Mobile Pico/Microcellular Networks" IEEE Communication Letters, Vol. 2, No. 7, July 1998. pp. 195-196.

[11] Ebersman H. G. and Tonguz O. K., "Handover ordering using signal prediction priority queuing in personal communication systems," IEEE Trans. Veh. Technol., vol. 48, Jan. 1999, pp. 20-35.

[12] Dharma P. Agrawal et al, "Performance Analysis of Handoff Schemes With Preemptive and Nonpreemptive Channel Borrowing in Integrated Wireless Cellular Networks" IEEE Transactions on Wireless Communications. Vol. 4, No. 3, May 2005. pp 1222-1233.

[13] Ming-Hui Jin et al, “An Intelligent Handover Scheme for the Next Generation Personal Communication Systems". Journal of Communications and Networks Vol. 6, No. 3, September 2004. pp 245-257.

[14] Onah, F. I. And Ani, C. I. "Application of Integrated modelling technique for data services resource allocation in ATM based private WAN", Nigerian Journal of Technology Vol. 33, No. 1, Jan, 2014, pp.72

[15] Jochen Schiller, Mobile Communications, second edition, Addison Wesley, 2003, page 117. 